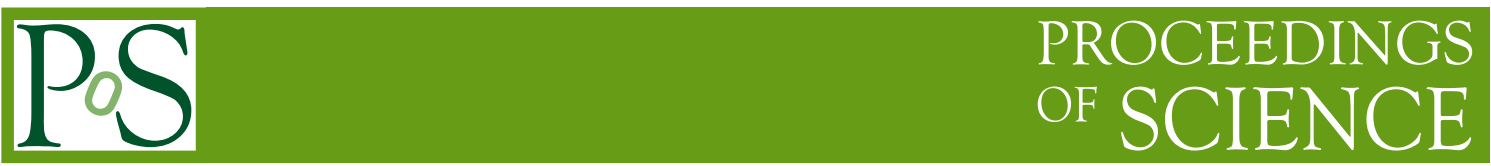

\title{
Solar Influence on Decay Rate (SIDR) Experiment
}

\author{
George Bashindzhagyan*, ${ }^{*}$ Virgil Barnes, ${ }^{b}$ Ephraim Fischbach, ${ }^{b}$ Gagik Hovsepyan, ${ }^{c}$ \\ Natalia Korotkova, ${ }^{a}$ Mikhail Merkin, ${ }^{a}$ Nikolai Sinev, ${ }^{d}$ Alexander Voronin ${ }^{a}$ \\ ${ }^{a}$ M.V. Lomonosov Moscow State University $(R U)$ \\ ${ }^{b}$ Purdue University (IN, USA) \\ ${ }^{c}$ Yerevan Physics Institute (AM) \\ ${ }^{d}$ University of Oregon (OR, USA) \\ E-mail: george.bashindzhagyanecern.ch
}

The goal of the proposed experiment is to check the evidence for a possible solar influence on nuclear decay rates, and to measure any effect quantitatively. Simultaneous decay rate measurements with many identical radioactive sources would allow us to study any possible correlations between their rate changes, and thus to improve the accuracy and reliability of the measurements. Positioning the sources with various distances between them (up to $2000 \mathrm{~km}$ ), and at different altitudes above sea level and underground, will help to determine which particles, rays and other outside influences can be responsible for nuclear decay rate changes (if they really exist).

The 34th International Cosmic Ray Conference,

30 July- 6 August, 2015

The Hague, The Netherlands

${ }^{*}$ Speaker. 


\section{Motivation}

Small periodic annual deviations of the data points from an exponential decay curve were observed in 1982-85 by D.E. Alburger et al. [1], when they measured the half-life of ${ }^{32} \mathrm{Si}$. Similar results were obtained with ${ }^{226} \mathrm{Ra}$ by H. Siegert et al. and presented in 1998 [2]. It's useful to point out that the goal of the mentioned experiments was to measure the half-life of the radiactive elements and the registered deviations were the side effects.

J.H.Jenkins, E. Fischbach et al. analyzed the data presented in [1,2] and found a correlation between decay rate and Earth-Sun distance (see Figures 1,2). They concluded that there is serious evidence of solar influence on decay rate [3]. Other experimental results confirming this conclusion have been also presented $[4,5]$.

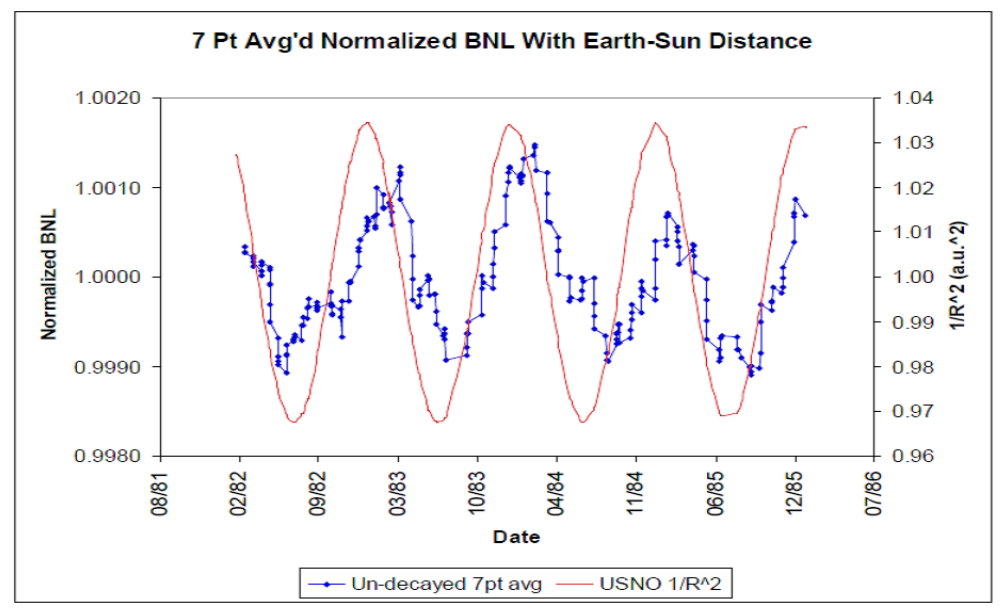

Figure 1: ${ }^{32} \mathrm{Si}$ decay rate deviations registered by D.E. Alburger et al. [1] (blue dots) and Earth-Sun distance changes (red curve) added by J.H. Jenkins, E. Fischbach et al. [3].

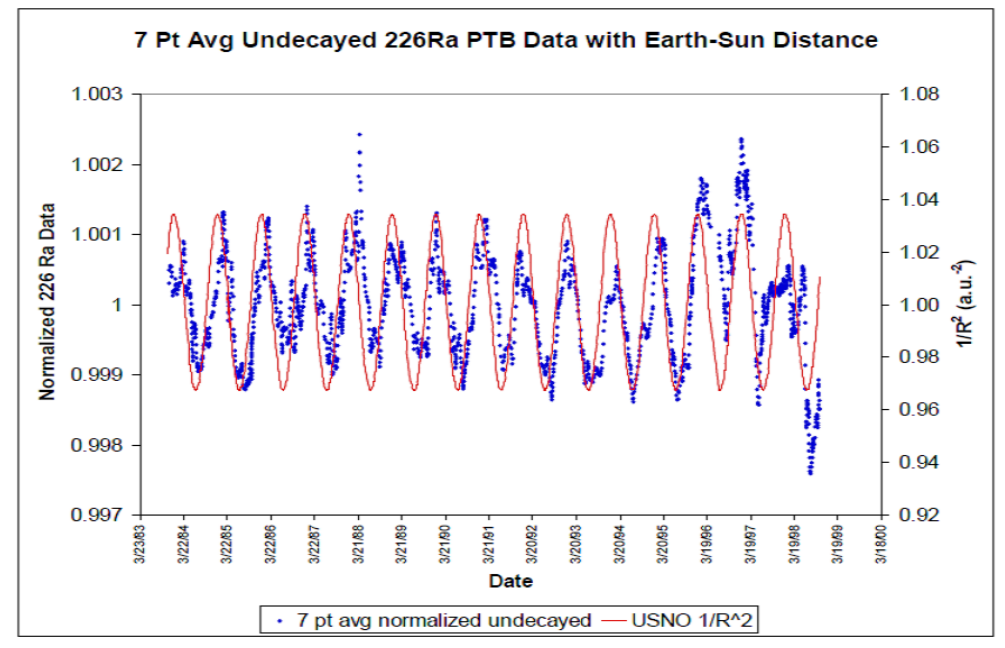

Figure 2: ${ }^{226}$ Ra decay rate deviations registered by H.Siegert et al. [2] (blue dots) and Earth-Sun distance changes (red curve) added by J.H. Jenkins, E. Fischbach et al. [3]. 


\section{Objectives}

The goals of the experiment are to check the evidences of radioactive decay rate changes and, if they are confirmed, to look for correlations between them and Sun-Earth distance as well as other external events, changes and effects.

It's well known that the major problem of decay rate change measurements is the rate of random fluctuations. But if there is an influence of outside factors like Sun-Earth distance changes, they have to be observed in many identical radioactive element samples simultaneously. Existence of observed correlated in time changes allows a significant decrease of the effect of random fluctuations, thus improving the accuracy of the measurements. The next step of the experiment is to look for correlations between the rate changes and various external events and processes, like SunEarth distance changes, solar flares and different local events like thunderstorms. If correlations between nearby modules are observed, it will be very interesting to check if there is a correlation between the modules separated by rather long distances like $2000 \mathrm{~km}$, on mountain level and deep underground.

Existence or absence of the correlations among many identical modules in very different natural conditions may help to understand the cause of any radioactive decay rate changes.

\section{Method}

Four identical modules will be used in Yerevan (Armenia) and three in Moscow (Russia). In Yerevan two modules will be placed nearby to study very local influences. A third module will be positioned in the Nor Amberd cosmic ray station $2000 \mathrm{~m}$ above sea level to pick up correlations separated by an average distance of about $40 \mathrm{~km}$ and different altitude. The fourth module will be at $235 \mathrm{~m}$ underground, or about $500 \mathrm{~m}$ water equivalent, to exclude cosmic ray influences except high energy muons and neutrinos. In Moscow two modules are also positioned nearby and the third one is about $20 \mathrm{~m}$ underground. The distance between Yerevan and Moscow will help to exclude local influences.

Each module has independent well stabilized power supply and temperature stabilization. It consists of a ${ }^{90} \mathrm{Sr}$ radioactive source, a silicon pad detector, front-end electronics and a counter. Start and stop commands for the counter are generated by an internal clock, but all module clocks are synchronized every 24 hours to achieve precise coincidence of start and stop commands inside all the modules. The counting period, initially assumed to be $1 \mathrm{~min}$., can be changed remotely, depending on current experimental results. When the counting period is over, the number of counted decays and the period ID are transmitted to cluster computers in Yerevan and Moscow correspondingly and accumulated there. Both clusters exchange information, allowing parallel data acquisition and preliminary analysis.

\section{References}

[1] D.E. Alburger, G. Harbottle and E.F. Norton, Half-life of ${ }^{32}$ Si, Earth and Planetary Science Letters 78 (1986) 168-176

[2] H. Siegert, H. Schrader and U. Schoetzig, Half-life Measurements of Europium Radionuclides and the long-term stability of Detectors, Appl. Radiat. Isot. 49 (1998) 1397-1401 
[3] J.H. Jenkins, E. Fischbach, J.B. Buncher, J.T. Gruenwald, D.E. Krause, J.J. Mattes, Evidence for Correlations Between Nuclear Decay Rates and Earth-Sun Distance, Astropart. Phys. 32 (2009) 42-46

[4] Yu.A. Baurov, Yu.G. Sobolev, V.F. Kushniruk, Yu.V. Ryabov, Experimental investigations of changes in the rate of beta decay of radioactive elements, Physics of Atomic Nuclei 70 (2007) N11 1825-1835

[5] J.H. Jenkins, E. Fischbach, Perturbation of Nuclear Decay Rates During the Solar Flare of 13 December 2006, Astropart. Phys. 31 (2009) 407-411 